$\mathrm{Ab}$ and cytokine levels in both FS-blood and serum from leprosy patients in South-Africa, Brazil, Bangladesh and the Netherlands and (their) contacts were measured using a portable reader.

Results Excellent correlation was demonstrated between data for anti-PGL-I IgM Ab and cytokines obtained with serum and FS blood from the same individuals.

Conclusion The quantitative UCP-LF test strips detecting antiPGL-I IgM Ab and cytokines for the detection of M. leprae infection is compatible with fingerstick blood allowing nearpatient testing and immediate appropriate follow-up counselling.

\section{OC 8259 THE VALIDATE NETWORK: EXPLOITING SYNERGIES BETWEEN COMPLEX INTRACELLULAR NEGLECTED PATHOGENS TO EXPEDITE VACCINE R\&D FOR TUBERCULOSIS, LEISHMANIASIS, LEPROSY AND MELIOIDOSIS}

${ }^{1}$ Samantha Vermaak*, ${ }^{2}$ Helen Fletcher, ${ }^{1}$ Helen McShane. ${ }^{1}$ The Jenner Institute, Nuffield Department of Clinical Medicine, University of Oxford, UK; ${ }^{2}$ The London School of Hygiene and Tropical Medicine, UK

\subsection{6/bmjgh-2019-EDC.5}

Background The VALIDATE 'VAccine deveLopment for complex Intracellular neglecteD pAThogEns' Network is a Global Challenges Research Fund (GCRF) Network, funded by the UK MRC and BBSRC and led by the University of Oxford and the London School of Hygiene and Tropical Medicine. It aims to accelerate vaccine development for four intracellular pathogens, Mycobacterium tuberculosis, Leishmania spp, Mycobacterium leprae and Burkholderia pseudomallei, by creating a network of scientists from around the world in an interactive community, sharing information, learning from synergies and differences, and forming new collaborations promoting crossdisciplinary, cross-pathogen, and cross-continent research.

Membership Currently VALIDATE has 125 members from 66 institutes in 28 countries, including world leading scientists, post-doctoral researchers, postgraduate students, and interested lay members from academia, governmental agencies, industry, and non-profits.

Activities VALIDATE has four activity streams: 1) providing funding to its members, including pump-priming grants for excellent research, training grants for early career researchers, and fellowships to transition post-doctoral researchers to independence, 2) a members' data-sharing portal, to encourage real-time sharing of data, catalysing the application of insights from one field into another, with an in-house Research Data Analyst working on cross-pathogen applications, 3) providing CPD opportunities for our members, including workshops, seminars and a mentoring scheme, and 4) speeding the dissemination of useful and relevant information via a hub website (www.validate-network.org) and social media (@NetworkValidate) where our members can easily find information about new research, relevant funding calls, events, and training, mentoring and other opportunities. Interested parties can read about our funded work, while a searchable directory of members on our website and a free annual meeting facilitates the formation of new collaborations.

VALIDATE is free to join and has an inclusive membership. This network would be of interest to researchers at the EDCTP Forum working on vaccine development for tuberculosis, leishmaniasis, leprosy and melioidosis.

\section{OC 8277 IDENTIFICATION OF NEW CEREBROSPINAL FLUID AND BLOOD-BASED BIOMARKERS FOR THE DIAGNOSIS OF TUBERCULOUS MENINGITIS IN CHILDREN}

Masilo Charles Manyelo*, Regan S Solomons, Gerhard Walzl, Novel N Chegou. Stellenbosch University, South Africa

\subsection{6/bmjgh-2019-EDC.6}

Background Tuberculous meningitis (TBM) is the most severe form of extrapulmonary tuberculosis (TB). It mostly affects young children and results in high morbidity and mortality, mainly due to diagnostic delay. There is an urgent need for new tests for the earlier and accurate diagnosis of the disease. We previously identified a 3-marker cerebrospinal fluid (CSF) biosignature (VEGF, IL-13, and LL-37) with potential to diagnose TBM. In the present study, we show that CSF and blood-based biosignatures may be useful in the diagnosis of TBM.

Methods CSF and serum samples were consecutively collected from 47 children that were admitted to the Tygerberg Academic Hospital in Cape Town, South Africa, on suspicion of having TBM. Using a multiplex platform, the concentrations of 69 host markers were evaluated in the CSF and serum samples from all the study participants, followed by statistical analysis to ascertain the usefulness of these biomarkers as diagnostic candidates for TBM disease.

Results Out of the 47 study participants, 23 (48.9\%) were finally diagnosed with TBM and $6(12.8 \%)$ were infected with HIV. Several CSF and serum biomarkers showed potential individually as diagnostic candidates for TBM as ascertained by area under the receiver operator characteristics curve (AUC). However, the main findings of our study were the identification of a four-marker CSF biosignature which diagnosed TBM with an AUC of 0.97 (95\% CI, 0.92-1.00), and a 3-marker serum biosignature which diagnosed TBM with an AUC of 0.84 (95\% CI, 0.73-0.96). We also validated a previously identified 3-marker CSF biosignature (VEGF, IL13 and LL37) in the study.

Conclusion CSF and serum biosignatures may be useful in the diagnosis of TBM in children. Our findings require further validation in larger, multi-site studies after which the biosignatures may be incorporated into point-of-care diagnostic tests for TBM.

\section{OC 8360 GLOBAL HEALTH RESEARCH AND ITS ROLE IN IMPROVING HEALTH AND HEALTH EQUITY IN AFRICA}

${ }^{1}$ Nchangwi Syntia Munung ${ }^{*},{ }^{1}$ Jantina De Vries, ${ }^{2}$ Bridget F Pratt. ${ }^{1}$ University of Cape Town, South Africa; ${ }^{2}$ Nossal Institute for Global Health, Melbourne School of Population and Global Health, Australia

\subsection{6/bmjgh-2019-EDC.7}

Background Health research has the potential to generate knowledge that may be used to improve health and health equity. This has led to calls for African governments to dedicate at least $2 \%$ of their national budgets to heath research, but such resource allocations have never been achieved. Rather, most of health research in Africa continues to be funded by high-income countries (HICs) and involves collaborative partnerships between researchers in high-income countries and those in Africa. These research partnerships have many benefits, but they also raise ethical issues related to justice and fairness in global health research. 
Methods The 'Research for Health Justice Framework' makes recommendations on how global health research partnerships may foster the ideals of justice through their selection of research populations and questions, research capacity strengthening, delivery of ancillary care and the provision of post-trial benefits. We applied these criteria to collaborative genomics research consortia in Africa (an example of global health research in Africa).

Results The results show that the lack of national health research priorities in most African countries hinders the intention of global health actors to use global health research as a means of promoting global health equity. Furthermore, capacity building efforts need to be more coordinated and monitored. The responsibility for this lies with several actors. Conclusion The potential for global health research to improve the health capability of countries in Africa will require that attention is paid to research that improves the health of people in Africa and that global health research partnerships identify, first and foremost, what kind of capacity strengthening is required and who is responsible for this activity. African governments and research institutions can play a role to help global health research improve health and health equity in Africa, in ways that are sustainable.

\section{OC 8378 REQUIREMENTS FOR THE ETHICAL CONDUCT OF CLINICAL RESEARCH IN CAMEROON, SUB-SAHARAN AFRICA: THE IMPORTANCE OF ACTIVE MONITORING}

${ }^{1}$ Marceline Djuidje Ngounoue*, ${ }^{2}$ Jerome Ateudjieu, ${ }^{1}$ Charles Fokunang, ${ }^{3}$ Primus Che Chi, ${ }^{1}$ Mireille Ndje Ndje, ${ }^{1}$ Sylvie Kwedi Nolna, ${ }^{4}$ Gisele Magne, ${ }^{5,6}$ Lazare Kaptue. ${ }^{1}$ University of Yaounde I, Cameroon; ${ }^{2}$ University of Dschang, Cameroon; ${ }^{3}$ Peace Research Institute Oslo (PRIO), Norway; ${ }^{4}$ Clinique Bastos, Cameroon; ${ }^{5}$ Universite des Montagnes, Bangangte, Cameroon; ${ }^{6}$ Cameroon National Ethics Committee, Cameroon

\subsection{6/bmjgh-2019-EDC.8}

Background Previous evaluation of the state of ethics regulation in Cameroon revealed: law regulating clinical research is lacking; existing committees lack infrastructure and financial support to sustainably review and effectively monitor approved protocols. The present Cameroon National Ethics Committee (CNEC)-EDCTP project aimed at implementing and evaluating active monitoring of clinical research in Cameroon.

Methods Between 2011-2013, approved clinical trials and protocols involving transfer of biological materials abroad were consecutively monitored. The monitoring tool, a questionnaire on the conformity of key documents e.g. research protocols, ethical clearance, informed consent documents, investigator's brochure, with a focus on GCP standards was sent to promoters/investigators ten days prior to the field visit. Teams of two-three monitors, made up of CNEC members and independent consultants, were mobilised per site (hospital/research institute/NGO). Reports with key recommendations were submitted to CNEC for review and approval, to different promoters/investigators, and the Regulatory Authority for action; the monitoring summary was submitted to EDCTP.

Results Up to 22 site visits were done throughout the country, monitoring about 30 protocols within 11 hospitals, 9 research institutes, a National Programme and a non-governmental organisation. All sites had ethical clearance and administrative authorisation for research. From the registered number of research participants, less than half of the signed consent forms were available. Other issues were the lack of full involvement of local investigators, inexistence of material transfer and data sharing agreements with collaborative studies, non-implication of study communities/participants. As educators-consultants, monitors formulated recommendations to investigators, ethics committees and regulatory authority, insisting on the implication of local PI/collaborators with defined percentages of time to be devoted for research and good participatory practice among research communities/participants. Conclusion Active monitoring shows some formality in the application of ethical/administrative clearance in Cameroon. However, complex issues raised confirm the necessity of continuous monitoring to meet the high standards for clinical research ethics in Cameroon.

\section{OC 8391 AFREENET (AFRICA ETHICS EXCELLENCE NETWORK): A NETWORK OF NATIONAL RESEARCH ETHICS COMMITTEES ENGAGED IN THE REINFORCEMENT OF THEIR CAPACITIES}

${ }^{1}$ Virginie Pirard, ${ }^{2}$ Louis Penali, ${ }^{4}$ Armande Gangbo, ${ }^{3}$ Oumou Younoussa Sow, ${ }^{1}$ Samira Ouchhi*. ${ }^{1}$ Institut Pasteur, Paris, France; ${ }^{2}$ National Ethics Committee for Health Research, Côte d'Ivoire; ${ }^{3}$ National Ethics Committee for Health Research, Conakry, Guinea; ${ }^{4}$ National Ethics Committee for Health Research, Benin

10.1136/bmjgh-2019-EDC.9

Background The establishment of research ethics committees (RECs) in charge of reviewing research protocols answers the need to regulate with an ethical framework the development of clinical trials, biomedical research and technologies affecting human health. The first RECs were instituted at the national level in the $60 \mathrm{~s}$, and were gradually put in place in Africa as a result of research projects development in the area of epidemics such as HIV infection, and to meet one of the major requirements of the Helsinki Declaration and the international ethical Guidelines CIOMS: 'each research protocol involving humans has to be reviewed by an independent ethics committee'. However, RECs in Africa are still facing various challenges in the accomplishment of their missions. Among them, RECs located in the West-African French speaking area are reporting an urgent need for networking and coordinating their effort.

Supported by EDCTP, AFREENET (AFRica Ethics Excellence NETwork) is a 3 year collaborative project between three RECs, respectively in Côte d'Ivoire, Guinea and Benin, and coordinated by the Ethics Unit of Institut Pasteur (France), which aims to reinforce RECs capacities.

The project will lead to reinforcement of training capacities through a long-term strategy to establish mechanisms to ensure and update members' training, and to develop a pool of ethics trainers. Based on RECs experiences, network activities will permit to share and identify valuable practices on global ethics oversight, such as monitoring, SOPs, regulatory issues and sensitisation. The preparation of Ethics Committees for the potential occurrence of outbreaks will be specifically addressed through an Outbreak preparation plan built upon lessons learnt from the Ebola crisis.

Creating synergies and mutual empowerment between African RECs will increase their visibility, their capacity for advocacy and their recognition as key actors of a responsible ethics research framework at the national, regional and international level. 